\title{
Interaction of erythrocytes in the process of pair aggregation in blood samples from patients with arterial hypertension and healthy donors: measurements with laser tweezers
}

\author{
Petr B. Ermolinskiy ${ }^{1 *}$, Andrei E. Lugovtsov ${ }^{1,2}$, Anastasiya I. Maslyanitsina ${ }^{1}$, Alexey N. Semenov ${ }^{1,2}$, \\ Larisa I. Dyachuk ${ }^{3}$, and Alexander V. Priezzhev ${ }^{1,2}$ \\ ${ }^{1}$ Department of Physics, \\ ${ }^{2}$ International Laser Center, and \\ ${ }^{3}$ Medical Research and Education Center of M.V. Lomonosov Moscow State University, Moscow, Russia. \\ * e-mail: peter.ermolinskiy@biomedphotonics.ru
}

\begin{abstract}
Two-channel laser tweezers are used to measure the interaction kinetics of two erythrocytes at the initial stage of aggregation, i.e., the formation of a pair aggregate in vitro. The study of erythrocytes interaction is important both for understanding the fundamental aggregation mechanisms and for evaluating the differences in kinetics and dynamics of aggregation, depending on the presence or absence of diseases that disturb the blood flow parameters and, therefore, the oxygen supply to tissues. We analyse the kinetics and dynamics of pair aggregation of erythrocytes in blood samples from more than 60 patients with arterial hypertension, as well as from a group of healthy donors. The results show that both kinetics and dynamics of erythrocyte aggregation are changed in the presence of the considered pathology. (C) 2018 Journal of Biomedical Photonics \& Engineering.
\end{abstract}

Keywords: aggregation; arterial hypertension; optical trap; manipulation of single cells; aggregation force; erythrocytes.

Paper \#3298 received 31 Aug 2018; revised manuscript received 16 Sep 2018; accepted for publication 19 Sep 2018; published online 30 Sep 2018. doi: 10.18287/JBPE18.04.030303.

\section{References}

1. A. Ashkin, J. M. Dziedzic, J. E. Bjorkholm, and S. Chu, "Observation of a single-beam gradient force optical trap for dielectric particles," Optics Letters 11(5), 288-290 (1986).

2. A. Ashkin, J. Dziedzic, "Optical trapping and manipulation of single living cells using infra-red laser beams," Berichte der Bunsen-Gesellschaft für Physikalische Chemie 98, 254-260 (1989).

3. A. Ashkin, Optical Trapping and Manipulation of Neutral Particles Using Lasers: a reprint volume with commentaries, World Scientific Publishing Co. Pte. Ltd., Singapore (2006).

4. K. Lee, A. V. Danilina, M. Kinnunen, A. V. Priezzhev, and I. Meglinski, "Probing the red blood cells aggregating force with optical tweezers," IEEE Journal on Selected Topics in Quantum Electronics 22(3), 7000106 (2016).

5. D. S. Moura, D. C. N. Silva, A. J. Williams, M. A. C. Bezerra, A. Fontes, and R. E. de Araujo, "Automatic real time evaluation of red blood cell elasticity by optical tweezers," Review of Scientific Instruments 86(5), 053702 (2015).

6. L. Yu, Y. He, A. Chiou, and Y. Sheng, "Deformation of biconcave Red Blood Cell in the Dual-beam Optical Tweezers," Proceedings of the 2011 COMSOL Conference, Boston (2011).

7. H. M. Nussenzveig, "Cell membrane biophysics with optical tweezers," European Biophysics Journal 47(5), 499-514 (2018). 
8. A. V. Korobtsov, S. Kotova, N. Losevsky, A. Mayorova, V. Patlan, E. Timchenko, N. Lysov, and E. Zarubina, "Optical tweezers technique for the study of red blood cells deformation ability," Laser Physics 22(7), 12651270 (2012).

9. S. Hu, D. Sun, “Automated transportation of single cells using robot-tweezer manipulation system," Journal of Laboratory Automation 16(4), 263-270 (2011).

10. T. Sugiura, S. Maeda, and A. Honda, "Pulse laser assisted optical tweezers for biomedical applications," 2012 Annual International Conference of the IEEE Engineering in Medicine and Biology Society, San Diego, CA, 4479-4481 (2012).

11. A. Y. Maklygin, A. V. Priezzhev, A. Karmenian, S. Y. Nikitin, I. S. Obolenskii, A. E. Lugovtsov, and K. Li, "Measurement of interaction forces between red blood cells in aggregates by optical tweezers," Quantum Electronics 42(6), 500-504 (2012).

12. V. V. Tuchin, "Fundamentals of laser biomedicine," Chap. 1 in Lasers and fiber optics in biomedical research, V.V. Tuchin (Ed.), 2nd edition, Fizmatlit, Moscow, 10-66 (2010) [in Russian].

13. A. V. Priezzhev, K. Lee, N. N. Firsov, and J. Lademann, "Optical Study of RBC Aggregation in Whole Blood Samples and Single Cells," Chap. 1 in Handbook on Optical Biomedical Diagnostics, V. V. Tuchin (Ed.), 2nd Edition, SPIE Press Bellingham, WA, United States (2016).

14. O. Baskurt, B. Neu, and H. Meiselman, Red Blood Cell Aggregation, CRC Press, Boca Raton, United States (2012).

15. S. Chien, L. A. Sung, S. Simchon, M. M. L. Lee, K.-m. Jan, and R. Skalak, "Energy balance in red cell interactions," Annals of the New York Academy of Sciences 416(1), 191-206 (1983).

16. K. Lee, C. Wagner, and A. V. Priezzhev, "Assessment of the "crossbridge"-induced interaction of red blood cells by optical trapping combined with microfluidics," Journal of Biomedical Optics 22(9), 091516 (2017)

17. K. Lee, A. Danilina, A. Potkin, M. Kinnunen, A. Priezzhev, and I. Meglinski, "RBC aggregation dynamics in autologous plasma and serum studied with two-channel optical tweezers," Proceedings of SPIE 9917, 991704 (2016).

18. K. Lee, A. Muravyov, A. Semenov, C. Wagner, A. Priezzhev, E. Lyubin, and A. Fedyanin, "Gamma globulins-induced interaction between two red blood cells: forces measurement with optical tweezers," Proceedings of SPIE 10336, 1033606 (2017).

19. J. Tripette, T. Alexy, M.-D. Hardy-Dessources, D. Mougenel, E. Beltan, T. Chalabi, R. Chout, M. EtienneJulan, O. Hue, H. J. Meiselman, and P. Connes, "Red blood cell aggregation, aggregate strength and oxygen transport potential of blood are abnormal in both homozygous sickle cell anemia and sickle-hemoglobin C disease," Haematologica 94(8), 1060-1065 (2009).

20. O. Baskurt, H. Meiselman, "Erythrocyte aggregation: basic aspects and clinical importance," Clinical Heomohreology and Microcirculation 53(1-2), 23-37 (2013).

21. E. Konstantinova, L. Ivanova, T. Tolstaya, and E. Mironova, "Rheological properties of blood and parameters of platelets aggregation in arterial hypertension," Clinical Hemorheology and Microcirculation 35(1-2), 135138 (2006).

22. M. D. Khokhlova, E. V. Lyubin, A. G. Zhdanov, A. A. Fedyanin, S. Y. Rykova, and I. A. Sokolova, "Normal and system lupus erythematosus red blood cell interactions studied by double trap optical tweezers: direct measurements of aggregation forces," Journal of Biomedical Optics 17(2), 025001 (2012).

23. G. Cicco, A. Pirrelli, "Red blood cell (RBC) deformability, RBC aggregability and tissue oxygenation in hypertension," Clinical Hemorheology and Microcirculation 21(3-4), 169-177 (1999).

24. B. Sandhagen, "Red cell fluidity in hypertension," Clinical Hemorheology and Microcirculation 21(3-4), 179181 (1999).

25. P. Gyawali, R. S. Richards, D. L. Hughes, and P. Tinley, "Erythrocyte aggregation and metabolic syndrome," Clinical Hemorheology and Microcirculation 57(1), 73-83 (2014).

26. P. Gyawali, R. S. Richards, and E. U. Nwose, "Erythrocyte morphology in metabolic syndrome," Expert Review of Hematology 5(5), 523-531 (2012).

27. E. Pytel, P. Duchnowicz, P. Jackowska, K. Wojdan, M. Koter-Michalak, and M. Broncel, "Disorders of erythrocyte structure and function in hypertensive patients," Medical Science Monitor 18(8), 331-336 (2012).

28. M. Kaczmarska, M. Fornal, F. H. Messerli, J. Korecki, T. Grodzicki, and K. Burda, "Erythrocyte membrane properties in patients with essential hypertension," Cell Biochemistry and Biophysics 67(3), 1089-1102 (2013).

29. J. Badlam, T. Bull, "Steps forward in the treatment of pulmonary arterial hypertension: latest developments and clinical opportunities," Therapeutic Advances in Chronic Disease 8(2-3), 47-64 (2017).

30. T. Nieminen, G. Knöner, N. R. Heckenberg, and H. Rubinsztein-Dunlop, "Physics of optical tweezers," Methods in Cell Biology 82, 207-36 (2007).

31. A. Gennerich, Optical Tweezers. Methods and Protocols, Humana Press (2017).

32. Video demonstrations of experiments with laser tweezers, obtained by P.B. Ermolinskiy, A.E. Lugovtsov, A.I. Maslyanitsina, A.N. Semenov, L.I. Dyachuk, A.V. Priezzhev. 
33. J. Lasch, G. Küllertz, and J. R. Opalka, "Separation of erythrocytes into age-related fractions by density or size? Counterflow centrifugation," Clinical Chemistry and Laboratory Medicine 38(7), 629-632 (2000).

34. M. Tavakol, O. Abouali, M. Yaghoubi, and G. Ahmadi, "Dispersion and Deposition of Ellipsoidal Particles in a Fully Developed Laminar Pipe Flow Using Non-Creeping Formulations for Hydrodynamic Forces and Torques," International Journal of Multiphase Flow 75, 54-67 (2015).

35. R. J. Fish, M. Neerman-Arbez, "Fibrinogen gene regulation," Thrombosis and Haemostasis 108(3), 419-26 (2012).

36. A. N. Semenov, A. E. Lugovtsov, K. W. Li, A. A. Fabrichnova, Y. A. Kovaleva and A.V. Priezzhev, "Applying methods of diffuse light scattering and optical trapping for assessing blood rheological parameters: erythrocytes aggregation in diabetes mellitus," Izvestie Saratov University (N.S.) 17(2), 85-97 (2017) [in Russian].

\section{Introduction}

Laser tweezers (LT) are devices that allow for trapping and manipulation of microobjects without direct mechanical contact with them. The principle of their operation is based on the interaction of a sharply focused laser beam with the trapped microobject. For the first time the possibility of trapping and manipulation of dielectric particles by means of a sharply focused laser beam was demonstrated by Arthur Ashkin in 70 s of the last century by the example of spherical microparticles in water, which finally led to the invention of LT [1]. They became a necessary tool in the solution of many problems of physics and biology $[2,3]$. For example, LT are used for living cells trapping and manipulation, sorting, measuring the cell elasticity and deformability $[4,5,6,7,8]$. LT are also used to study the cells cytoskeletons, to measure viscosity and elasticity of biopolymers [3]. It is also possible to measure the forces of interaction between living cells, in particular, the red blood cells [4]. The field of LT applications is permanently expanding.

To date different schemes of LT have been designed, aimed at solving different research and technical problems. For example, there are setups that allow automated trapping and manipulation of single cells [9]. Alongside with continuous-wave lasers, pulsed lasers are also applied in LT schemes in order to improve the trapping efficiency and manipulating velocity [10]. Using a circularly polarised trapping beam, one can rotate the trapped cell [3]. The character of trapping depends on the beam profile [3]. In our experiments we used two-channel laser tweezers based on the continuous-wave lasers with Gaussian beam profile to measure the kinetic and dynamic parameters of erythrocytes interaction in the process of pair aggregation (see Materials and methods).

When the laser beam traps a living cell, it is important to monitor the influence of the optical trapping on the cell properties, since the capture can affect the linear dimensions, shape and orientation of the cell depending on the elasticity of its membrane and the trapping force [11]. The laser radiation wavelength should be chosen in the range of maximum optical transparency of the studied object, thus eliminating the thermal effect, i.e., the object heating in the process of investigation [12].

The interaction of erythrocytes with each other leading to the formation of aggregates is one of the most important mechanisms regulating the fluidity of blood in vessels. This interaction is referred to as aggregation, and it essentially affects the blood microcirculation and rheology of blood in the entire organism [13]. In the vessels in vivo the aggregation of erythrocytes is a spontaneous and reversible process, since under the effect of shear stresses that arise in the blood flow the aggregates are separated into individual cells, which can aggregate again as a result of subsequent interaction. To this day the erythrocytes aggregation mechanism has not been fully studied [14]. The interaction of aggregating erythrocytes is commonly described using two models differing in the definition of forces responsible for aggregate formation. In the model of depletion layer the interaction is described by osmotic forces that arise in the solutions of macromolecules surrounding the cells, e.g., in blood plasma [14]. In the model of bridges, the interaction is described by the forces that arise due to adsorption of macromolecules at the surface of membranes of adjacent erythrocytes [15]. To date there are serious arguments in favour of both models $[14,16]$. However, making a final decision on the correctness of model considerations requires additional studies.

The processes of aggregation and disaggregation of erythrocytes can be described by a set of parameters, the meaning of which depends on the method of measurement, in particular, on whether the parameters are measured in a sample of large ensemble of cells, e.g., in whole blood, or in a sample of single erythrocytes (highly diluted suspension of single cells). The values of these parameters depend on the composition of the medium surrounding the erythrocytes. In the organism (in vivo) it is the blood plasma, but out of the organism (in vitro) the composition of the environment can be purposely changed for the sake of study. In particular, the measurements are performed in blood serum (purified plasma free of fibrinogen protein, which is one of the main proaggregants) [17] or in solutions of 
macromolecules affecting the processes of blood cells interaction in a certain way [18].

When such parameters of aggregation as the aggregation rate and the hydrodynamic strength of aggregates differ from the normal values to higher values, the viscosity of blood increases that decelerates the blood flow and disturbs microcirculation in the organism. The abnormal aggregation of erythrocytes was observed by a number of researchers under the diabetes mellitus, arterial hypertension, cardiovascular pathologies, cerebrovascular insufficiency [19, 20, 21]. In Ref. [22] the alterations of such aggregation parameters of erythrocytes as the interaction force and the aggregation rate from the norm in patients with systemic lupus erythematosus were reported. Most researchers performed measurements of microrheological parameters of erythrocytes (i.e., the parameters of cell aggregation and deformability) with samples of whole blood, i.e., large ensembles of particles, using the methods of ectacytometry and diffuse light scattering [13]. In the present study, the changes of cell aggregation parameters (aggregation time and interaction forces) were investigated for pairs of single blood cells from patients with arterial hypertension. In the study of interaction between single erythrocytes, the introduced aggregation parameters are different from those in a large cell ensemble, since in the latter case the measured values are averaged over all cells in the ensemble.

Arterial hypertension (AH) is a chronic disease characterized by persistently high arterial pressure. Under this disease the microrheological properties of blood become changed, which essentially affects the blood flow in the vessels [23, 24, 25]. Usually, the patients with $\mathrm{AH}$ suffer from concomitant diseases, e.g., in the case of metabolic syndrome [26]. To date the results are obtained that evidence in favour of the presence of oxidative balance disturbance in erythrocytes of patients with AH [27], which may cause hypoxemia and, in turn, further increase of the arterial pressure [28]. At the present new methods of $\mathrm{AH}$ treatment by correcting the aggregation state of erythrocytes are being developed [29]. Naturally, in this case the methods are required capable of quantitative assessment and monitoring of this state not only at the level of an ensemble of cells, but also at the level of interaction of individual erythrocytes. Thus, by studying the process of red blood cells aggregation, its origin and the factors that affect it, we approach the understanding of the aggregation mechanism, which in the future may give rise to novel methods of treatment of $\mathrm{AH}$ and other socially significant diseases.

The aim of the present work is to study the kinetics and dynamics of interaction of pair-aggregating erythrocytes using the laser tweezers in the blood samples from a group of patients with $\mathrm{AH}$ and a control group of healthy donors. The obtained data are statistically analysed and systemised; the values of the measured parameters for the two groups are compared.

\section{Experimental setup}

In this work, we used the two-channel homemade laser tweezers. A schematic layout of the setup is presented in Fig. 1. The basic elements are two diode-pumped Nd:YAG lasers (Shanghai Dream Lasers Technology) with the wavelength $1064 \mathrm{~nm}$ and the output power 200 $\mathrm{mW}$, and the water-immersion objective OLYMPUS with high numerical aperture (NA $=1.00$ ). The wavelength of the lasers is in the range of optical transparency of haemoglobin, which is the main component of an erythrocyte [12]. This fact allows elimination of heating effect of the trapping beam on the blood cells, located under the cover glass in a cuvette filled with saline solution. The power of the beams varied within the range $20-30 \mathrm{~mW}$. Earlier we have shown [11] that in such cuvette the temperature of a cell increases by $\sim 1{ }^{\circ} \mathrm{C}$ per each $10 \mathrm{~mW}$ of laser radiation power during the trapping time about 5-10 minutes. Therefore, in our experiments the temperature of cells could differ from that of the environment by less than $3{ }^{\circ} \mathrm{C}$, which lies within the limits of physiologic variations. Since the experiment with a pair of erythrocytes takes the time of 1-2 minutes, such thermal effect does not lead to essential changes of the studied parameters. The video images of interacting erythrocytes during every measurement were recorded using the CMOS camera DCC1645C (Thorlabs).

In the setup, two laser beams allow trapping and manipulation of two initially not interacting blood cells, drawing them together until point contact, overlapping their membranes. Typically, one of the beams is immobile and possesses greater trapping force than the other mobile one, whose position is varied by means of a controlled mirror. This facilitates a fixed position of erythrocyte in the immobile trap and direct measurement of interaction forces between the erythrocytes by means of moving the mobile beam. For example, in the case of membrane contact of the trapped erythrocytes under the gradual decrease of the beam powers and, therefore, trapping forces, at a certain moment of time the aggregation of the erythrocytes will occur, and the aggregated erythrocyte will move to the immobile beam trap region. Using a beam expander in this setup allows increasing the trapping gradient force, since by increasing the beam aperture we increase the convergence angle of the laser beam in the focal point of the objective, which increases the intensity gradient in the beam waist region [30]. In the setup, the power of each trap was variable by means of two polarisers, which, in turn, allowed controlling the trapping force. The physical principle of optical trapping formation and the basic components of laser tweezers are described in more detail in Ref. [31].

\section{Materials and methods of measurements}

The Medical Research and Education Centre of M.V. Lomonosov Moscow State University provided the blood samples used in our work after getting the informed consent of each patient. All experiments were 
performed during 5 hours from the moment of blood sampling. The sample used for measurements with LT was a suspension of erythrocytes, diluted by the autologous erythrocyte-depleted plasma, which was obtained as follows. The patient's whole blood samples were centrifuged (Eppendorf MiniSpin, Germany) at $180 \times \mathrm{g}$ for 10 minutes. The sediment erythrocytes were extracted from the plasma with micropipette, and the rest of the plasma was centrifuged at $3000 \times \mathrm{g}$ during 10 minutes for sedimentation of thrombocytes. After the separation of plasma from the sediment thrombocytes, the erythrocytes were diluted in the thrombocyte-free plasma at a ratio 1:1000. Such dilution is needed because in the study of single cells interaction the distance separating the adjacent non-interacting cells should be large enough not to affect the measurement process. The prepared sample was placed in the cuvette formed by the object glass slide and the cover one, the spacing between them being of the order of $100 \mu \mathrm{m}$ (Fig. 2).

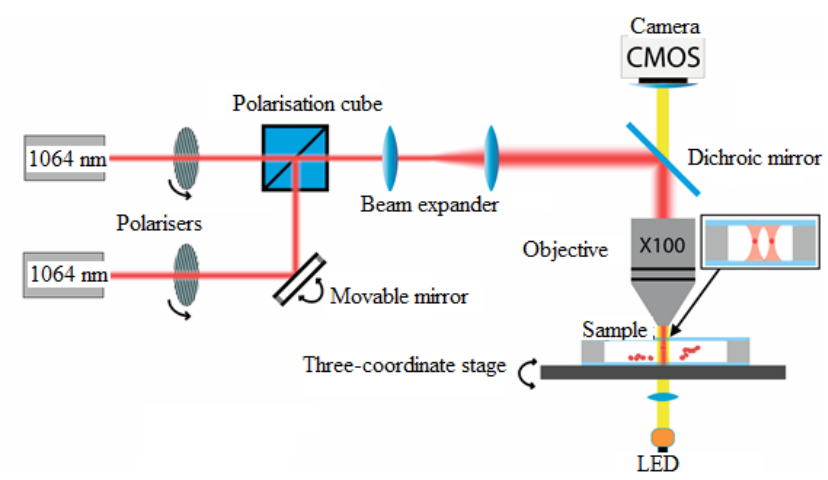

Fig. 1 Schematic diagram of the two-channel laser tweezers.

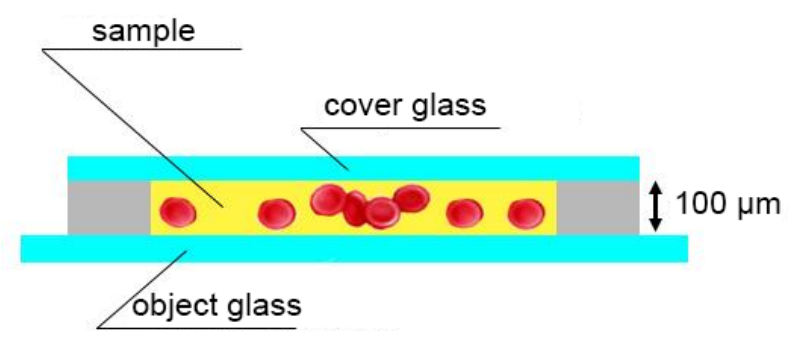

Fig. 2 Schematic diagram of blood sample cuvette.

After placing the cuvette with erythrocytes under the objective, objective focusing, and searching for two initially non-interacting erythrocytes they were sequentially captured by two traps, brought together till the contact by moving the mobile trap, and subjected to measurements. Three parameters were measured in the experiments, namely, the erythrocytes aggregation time (i.e., the time of their stacking upon each other resulting in the formation of a pair aggregate), the aggregation force (AF) and the disaggregation force (DF) (Fig. 3). The video demonstrations of the measurement of these parameters are available at [32].
The time of erythrocyte aggregation is the time interval from the contact of their membranes to the formation of a pair aggregate. Two erythrocytes are brought together until the state of a "point contact", i.e., the minimal overlap allowed by the setup [32]. Then the laser beams that form the traps are shut off and the spontaneous aggregation of erythrocytes by stacking of the cells upon each other is observed.

The aggregation force is the minimal force of optical trapping necessary to prevent spontaneous aggregation of two interacting cells. In this case, the surfaces of two erythrocytes are brought to overlapping using the LT by nearly $40 \%$, which is controlled visually. We choose the degree of overlapping phenomenologically as the most suitable for performing the experiments. Then the trapping force of one of the traps is gradually reduced until this force can prevent the beginning of the spontaneous aggregation of the cells.

The disaggregation force is the minimal force required to separate the pair of aggregated erythrocytes. In this case two interacting erythrocytes overlap by nearly $40 \%$ of their surface (which is controlled visually), and the mobile trap is displaced trying to separate the cells. If the separation is successful, the two erythrocytes are returned to the initial position $(40 \%$ overlap of membranes), the trapping force is slightly reduced, and the preceding steps are repeated until the force is unable to prevent the spontaneous aggregation of two cells. We chose the initial degree of overlap of the cells for measuring the disaggregation force phenomenologically as the most suitable for performing the experiments.

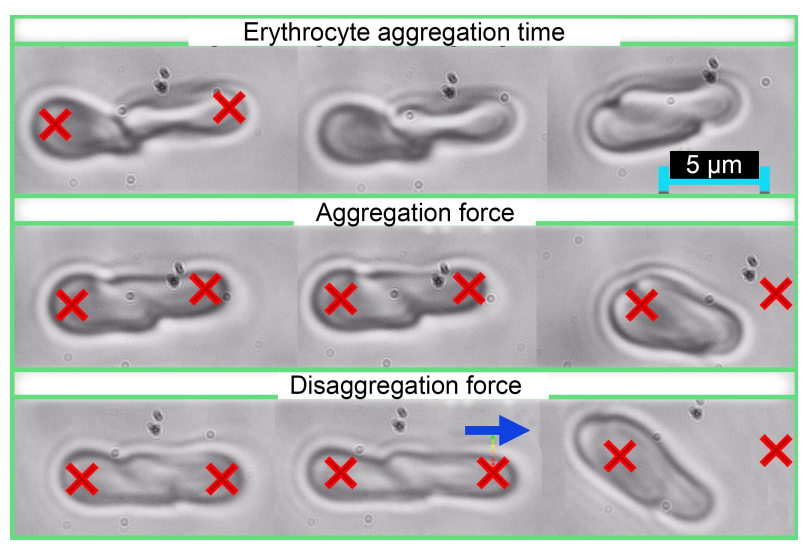

Fig. 3 Photo illustration of the processes of measuring the aggregation time, aggregation force, and disaggregation force of erythrocytes. Red crosses denote the positions where the cells were optically trapped.

The erythrocytes of human blood are different in age, size, and aggregation properties [33]. Therefore, for each blood sample the results were averaged, namely, the aggregation time was measured in 10 pairs of cells, and the AF and DF were measured in 5 pairs. All results were statistically analysed using the Statistica 13 software, and the data of donors from the control group and the patients with $\mathrm{AH}$ were compared using the standard Student's t-test for independent data samples. 
The typical statistical spread of values in the measurements of AF and DF for one patient due to the difference of cells did not exceed $10 \%$.

For measuring the dynamical characteristics of interaction of single cells, namely, the forces, the laser tweezers were calibrated by comparing the optical trapping force at a definite power of the laser beam with the viscous friction force (Stokes drag), acting on a trapped erythrocyte in a flow (Fig. 4). For this purpose, the erythrocyte was trapped in one channel of the laser tweezers and a flow of solvent solution was created with respect to it by moving the object stage with the cuvette. In the process of gradual increase of the flow velocity, a moment comes when the trapping force becomes smaller than the force of viscous friction, and the erythrocyte affected by the latter is released from the trap. Under the effect of the viscous friction force, the erythrocyte is deformed (elongated) becoming spheroidlike. Therefore, for the calculation of this force we modelled the erythrocyte by a spheroid, the form factor for which is presented in Ref. [34]. The video demonstration of the laser tweezers calibration is available at [32]. Thus, we obtain the experimental dependence of the viscous friction force upon the power of the laser beam. In this consideration, the viscous friction force coincides with the trapping force, since these forces are balanced when the erythrocyte is released from the trap. An example of the calibration curve is presented in Fig. 5.

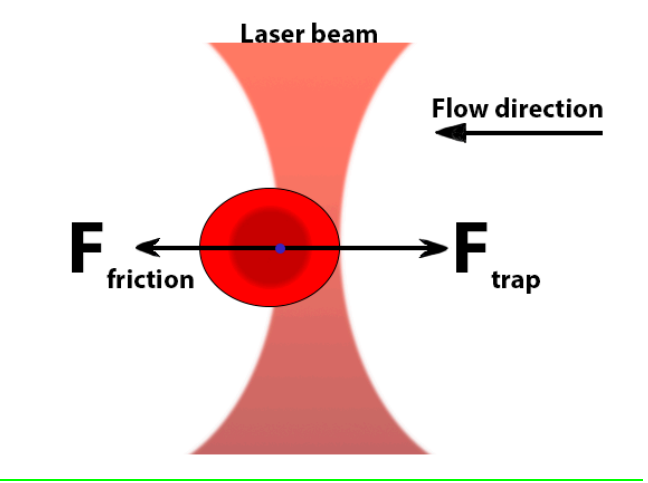

Fig. 4 Schematic comment to the process of laser tweezers calibration.

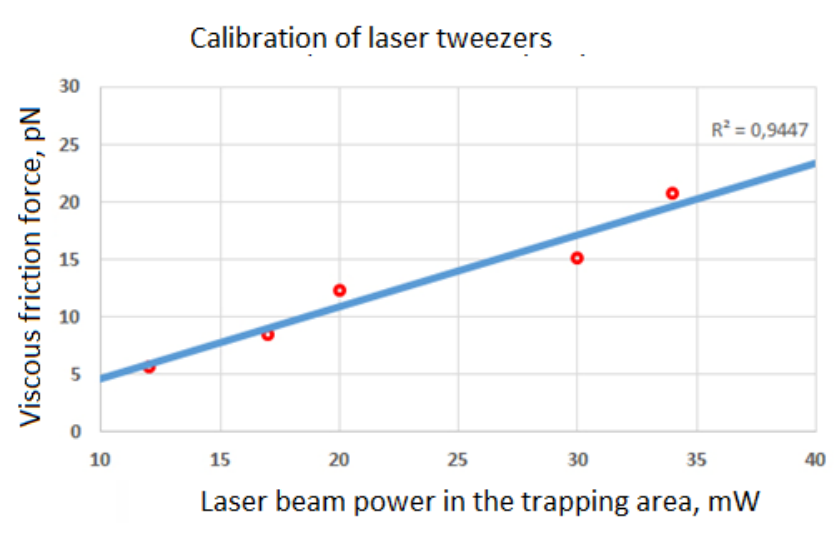

Fig. 5 Calibration curve for the laser tweezers.

\section{Results and discussion}

The erythrocyte aggregation parameters were measured in blood samples of 67 patients with $\mathrm{AH}$ aged $62 \pm 12$ and 8 donors of the control group (without $\mathrm{AH}$ and any other chronic diseases) aged $57 \pm 9$. The results are presented in Fig. 6. One can conclude that in the blood of patients the erythrocytes aggregate faster than in the blood of donors from the control group. In the case of $\mathrm{AH}$ the aggregation time is smaller by the statistically significant value of $24 \pm 3 \%$. In other words, during the same time more aggregates are produced in the blood of the patients with $\mathrm{AH}$, which leads to the increased viscosity of blood and, therefore, to the disturbance of blood microcirculation. No statistically significant difference in the erythrocyte aggregation force measured in the blood of patients with $\mathrm{AH}$ and healthy donors of the control group was detected, whereas the disaggregation force is by $28 \pm 5 \%$ greater in the patients with $\mathrm{AH}$ than in the donors of the control group, which is a statistically significant difference $(p<0.05)$. Therefore, in people suffering from $\mathrm{AH}$ the dispersion of erythrocytes requires higher shear stresses, than in healthy people.

The content of blood proteins in the patients with $\mathrm{AH}$ is presented in Table 1.

Table 1 Results of blood biochemical analysis in patients with $\mathrm{AH}$ in comparison with the norm limits. The norm limits are adopted from Refs. [35].

\begin{tabular}{lll}
\hline & AH & Norm \\
\hline Fibrinogen, g/l & $3.8 \pm 0.5$ & From 1.5 to 3.5 \\
\hline Total protein, g/1 & $70 \pm 4$ & From 60 to 83 \\
\hline Albumin, g/1 & $43 \pm 2$ & From 38 to 50 \\
\hline
\end{tabular}

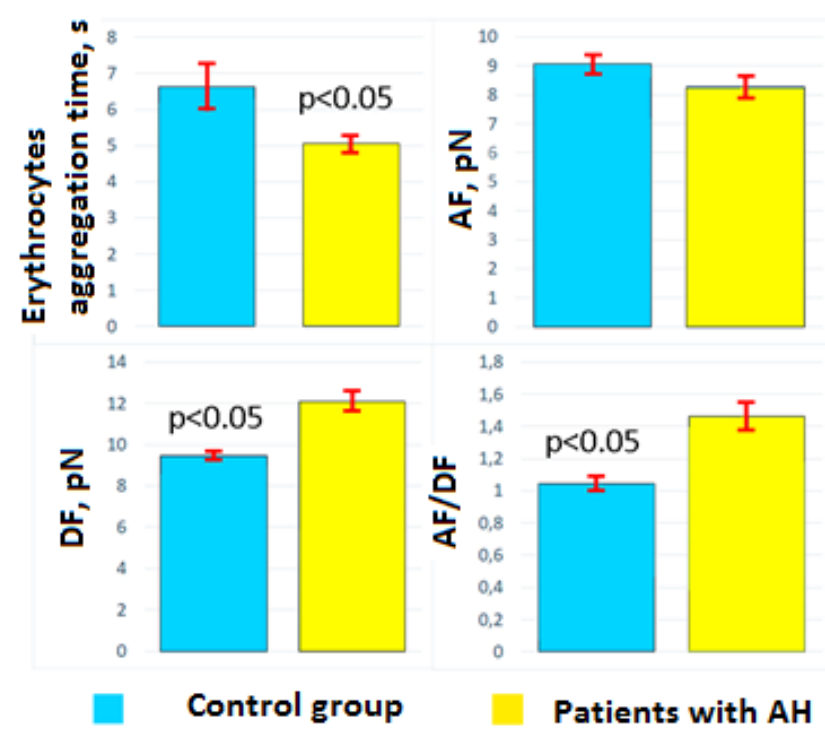

Fig. 6 Aggregation parameters: erythrocytes aggregation time, AF, DF and AF/DF for the patients with $\mathrm{AH}$ and the control group. The vertical bars show the mean-rootsquare error of mean values. 

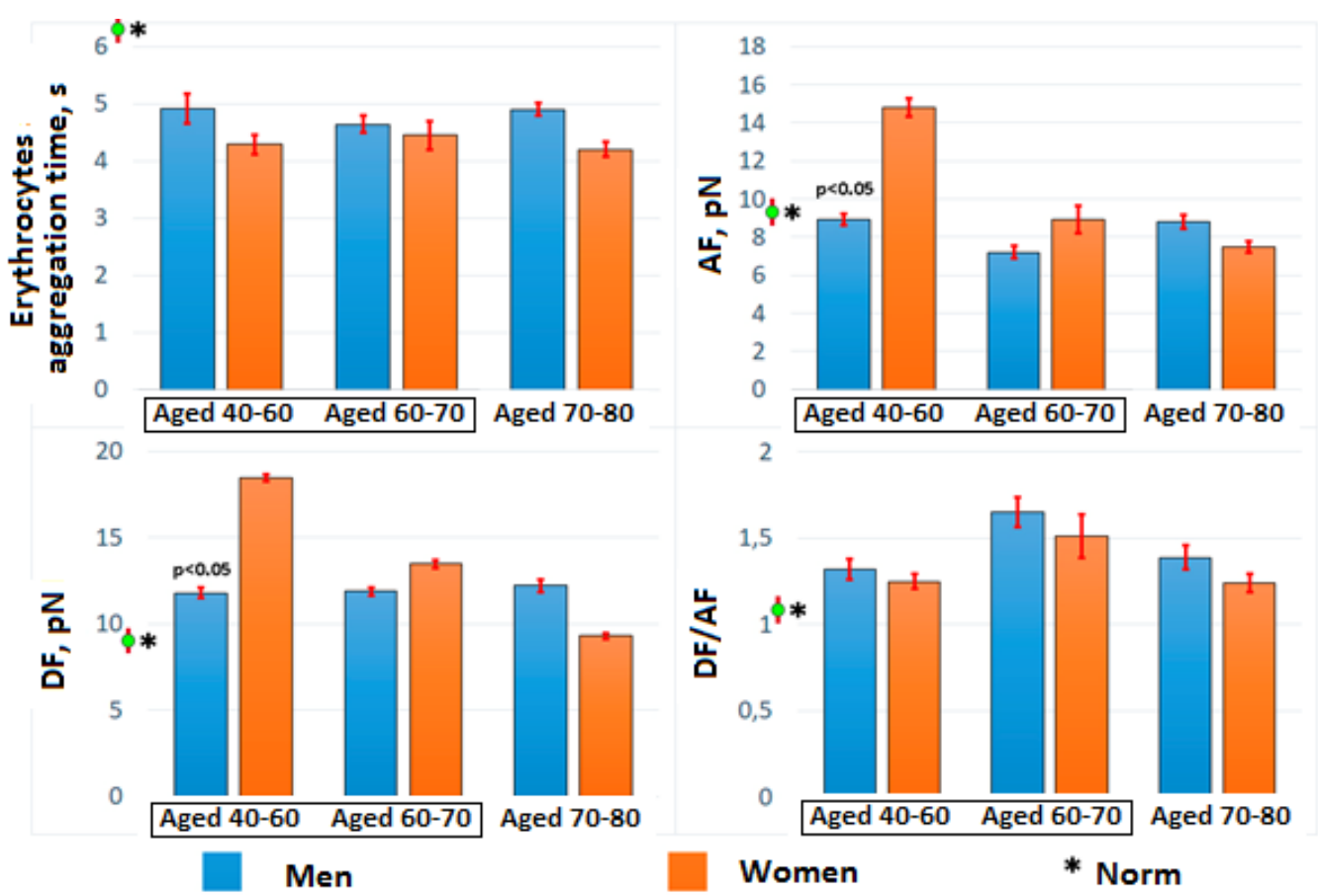

Fig. 7 Aggregation parameters: the erythrocytes aggregation time, AF, DF and the ratio DF/AF in men and women with $\mathrm{AH}$ for three age groups. Vertical bars indicate the mean-root-square error of mean values.

In patients with $\mathrm{AH}$ the concentration of fibrinogen typically corresponds to the upper limit of the norm (Table 1). Since the fibrinogen macromolecules are proaggregants, the enhanced aggregation of cells due to the increased concentration of this protein in the blood plasma of people with AH is expected.

The exact values of AF and DF can be different for the erythrocytes of different persons. However, our data show that the ratio of DF to AF can be more informative and stable than the parameters of the forces considered separately [36]. Thus, according to our data in the case of $\mathrm{AH}$ the DF/AF ratio amounts to nearly 1.5 , while for the control group it is about 1.0. In future, the DF/AF ratio may become a new diagnostic indicator of pathology.

Fig. 7 presents the distributions of aggregation parameters for male and female patients with $\mathrm{AH}$ aged in ranges of 40-60, 60-70 and 70-80. Since in the latter group due to the age the patients could suffer from concomitant diseases and purely age-related changes, in our case this group carries less reliable information. For healthy donors the values of the measured parameters are shown at the ordinate axis (with green point), because due to the smallness of the sample $(\mathrm{N}=8)$ their age distribution appears to be not correct. In the first two age groups, the aggregation time difference between male and female is statistically small. AF and DF in this sample monotonically decrease with age in women and demonstrates statistically significant difference from the same parameters in men. Within one age group the DF/AF ratio remains almost unchanged, however, between the age groups it differs with statistical significance $(\mathrm{p}<0.05)$.

\section{Conclusions}

The results of the measurements of erythrocyte aggregation parameters performed by means of laser tweezers according to the protocols developed by us have shown that such parameters as the cell aggregation time, disaggregation force, aggregation force, and the ratio of disaggregation to aggregation force in patients with arterial hypertension ( $\mathrm{AH})$ differ from those in the persons from the control group. The difference of aggregation parameters between two groups may be due to the pathological changes of both the properties of erythrocytes themselves, their morphology, and the composition of plasma in which they are suspended. Except fibrinogen, one of the main proaggregants, the concentration of which in patients with $\mathrm{AH}$ is somewhat above the range of norm, the protein composition of plasm of the majority of patients with $\mathrm{AH}$ does not leave the limits of norm. This may be an evidence of the dominating effect of the properties inherent in erythrocytes themselves (mechanical properties of the membrane and viscosity of cytosol) on the aggregation parameters of erythrocytes.

In men and women with arterial hypertension within one age group, the aggregation force and the disaggregation force statistically differ, while the disaggregation to aggregation force ratio remains within the error limits. Further acquisition of statistical data is expected to show whether the disaggregation to aggregation force ratio can be a reliable indicator of blood condition in patients with arterial hypertension. 


\section{Disclosures}

The authors have no relevant financial interests in this article and no potential conflicts of interest to disclose.

\section{Acknowledgments}

The authors are grateful to Dr. Kisung Lee for fruitful discussions during the experiments and preparing the paper.

The work was carried out under the financial support from the Russian Scientific Foundation (grant No. 1815-00422). 